\title{
Mixed monotone operator methods for the existence and uniqueness of positive solutions to Riemann-Liouville fractional differential equation boundary value problems
}

\section{Chengbo Zhai* and Mengru Hao}

"Correspondence: cbzhai215@sohu.com School of Mathematical Sciences, Shanxi University, Taiyuan, Shanxi 030006, P.R. China

\section{Abstract}

This work is concerned with the existence and uniqueness of positive solutions for the following fractional boundary value problem:

$$
\left\{\begin{array}{l}
-D_{0^{+}}^{v} y(t)=f(t, y(t), y(t))+g(t, y(t)), \quad 0<t<1, n-1<v \leq n \\
y^{(i)}(0)=0, \quad 0 \leq i \leq n-2 \\
{\left[D_{0^{+}}^{\alpha} y(t)\right]_{t=1}=0, \quad 1 \leq \alpha \leq n-2}
\end{array}\right.
$$

where $D_{0^{+}}^{v}$ is the standard Riemann-Liouville fractional derivative of order $v$, and $n \in N, n>3$. Our analysis relies on two new fixed point theorems for mixed monotone operators with perturbation. Our results can not only guarantee the existence of a unique positive solution, but also be applied to construct an iterative scheme for approximating it. An example is given to illustrate the main result.

MSC: 26A33; 34B18; 34B27

Keywords: Riemann-Liouville fractional derivative; fractional differential equation; positive solution; existence and uniqueness; fixed point theorem for mixed monotone operator

\section{Introduction}

In this paper, we investigate the existence and uniqueness of positive solutions for the fractional boundary value problem (FBVP for short) of the form:

$$
\left\{\begin{array}{l}
-D_{0^{+}}^{v} y(t)=f(t, y(t), y(t))+g(t, y(t)), \quad 0<t<1, n-1<v \leq n \\
y^{(i)}(0)=0, \quad 0 \leq i \leq n-2 \\
{\left[D_{0^{+}}^{\alpha} y(t)\right]_{t=1}=0, \quad 1 \leq \alpha \leq n-2}
\end{array}\right.
$$

where $D_{0^{+}}^{v}$ is the standard Riemann-Liouville fractional derivative of order $v$, and $n \in N$, $n>3$.

(0) 2013 Zhai and Hao; licensee Springer. This is an Open Access article distributed under the terms of the Creative Commons Attribution License (http://creativecommons.org/licenses/by/2.0), which permits unrestricted use, distribution, and reproduction in any medium, provided the original work is properly cited. 
Fractional differential equations arise in many fields such as physics, mechanics, chemistry, economics, engineering and biological sciences, etc.; see [1-6] for example. In the recent years, there has been a significant development in ordinary and partial differential equations involving fractional derivatives; see the monographs of Miller and Ross [3], Podlubny [5], Kilbas et al. [6], and the papers [7-16] and the references therein. In these papers, many authors have investigated the existence of positive solutions for nonlinear fractional differential equation boundary value problems. On the other hand, the uniqueness of positive solutions for nonlinear fractional differential equation boundary value problems has been studied by some authors; see $[10,14,17]$ for example.

In [18], Goodrich utilized the Krasnoselskii's fixed point theorem to study a FBVP of the form:

$$
\left\{\begin{array}{l}
-D_{0^{+}}^{v} y(t)=f(t, y(t)), \quad 0<t<1, n-1<v \leq n \\
y^{(i)}(0)=0, \quad 0 \leq i \leq n-2 \\
{\left[D_{0^{+}}^{\alpha} y(t)\right]_{t=1}=0, \quad 1 \leq \alpha \leq n-2}
\end{array}\right.
$$

and established the existence of at least one positive solution for FBVP (1.2). By using the same fixed point theorem, Goodrich [19] considered the existence of a positive solution to the following systems of differential equations of fractional order:

$$
\left\{\begin{array}{l}
-D_{0^{+}}^{\nu_{1}} y_{1}(t)=\lambda_{1} a_{1}(t) f\left(y_{1}(t), y_{2}(t)\right) \\
-D_{0^{+}}^{\nu_{2}} y_{2}(t)=\lambda_{2} a_{2}(t) g\left(y_{1}(t), y_{2}(t)\right)
\end{array}\right.
$$

where $t \in(0,1), v_{1}, v_{2} \in(n-1, n]$ for $n>3$ and $n \in N$, and $\lambda_{1}, \lambda_{2}>0$, with the following boundary value conditions:

$$
\begin{aligned}
& y_{1}^{(i)}(0)=0=y_{2}^{(i)}(0), \quad 0 \leq i \leq n-2, \\
& {\left[D_{0+}^{\alpha} y_{1}(t)\right]_{t=1}=0=\left[D_{0+}^{\alpha} y_{2}(t)\right]_{t=1}, \quad 1 \leq \alpha \leq n-2,}
\end{aligned}
$$

under the assumptions that $a_{1}, a_{2}, f, g$ are nonnegative and continuous. But the uniqueness of positive solutions is not treated in these papers.

Different from the works mentioned above, motivated by the work [20], we will use two fixed point theorems for mixed monotone operators with perturbation to show the existence and uniqueness of positive solutions for FBVP (1.1). To our knowledge, there are still very few to utilize the fixed point results on mixed monotone operators with perturbation to study the existence and uniqueness of a positive solution for nonlinear fractional differential equation boundary value problems. So, it is worthwhile to investigate FBVP (1.1) by using our new fixed point theorems in [20]. Our results can not only guarantee the existence of a unique positive solution, but also be applied to construct an iterative scheme for approximating it.

With this context in mind, the outline of this paper is as follows. In Section 2 we recall certain results from the theory of fractional calculus and some definitions, notations and results of mixed monotone operators. In Section 3 we provide some conditions, under which the problem FBVP (1.1) has a unique positive solution. Finally, in Section 4, we provide an example, which explicates the applicability of our result. 


\section{Preliminaries}

For the convenience of the reader, we present here some definitions, lemmas and basic results that will be used in the proofs of our theorems.

Definition 2.1 (See [18]) Let $v>0$ with $v \in R$. Suppose that $y:[a,+\infty) \rightarrow R$. Then the $v$ th Riemann-Liouville fractional integral is defined to be

$$
D_{a^{+}}^{-v} y(t):=\frac{1}{\Gamma(v)} \int_{a}^{t} y(s)(t-s)^{\nu-1} d s
$$

whenever the right-hand side is defined. Similarly, with $v>0$ and $v \in R$, we define the $v$ th Riemann-Liouville fractional derivative to be

$$
D_{a^{+}}^{v} y(t):=\frac{1}{\Gamma(n-v)} \frac{d^{n}}{d t^{n}} \int_{a}^{t} \frac{y(s)}{(t-s)^{v+1-n}} d s,
$$

where $n \in N$ is the unique positive integer satisfying $n-1 \leq v<n$ and $t>a$.

Lemma 2.2 (See [19]) Let $g \in C[0,1]$ be given. Then the unique solution to problem $-D_{0^{+}}^{v} y(t)=g(t)$ together with the boundary conditions $y^{(i)}(0)=0=\left[D_{0^{+}}^{\alpha} y(t)\right]_{t=1}$, where $1 \leq \alpha \leq n-2$ and $0 \leq i \leq n-2$, is

$$
y(t)=\int_{0}^{1} G(t, s) g(s) d s,
$$

where

$$
G(t, s)=\left\{\begin{array}{l}
\frac{t^{\nu-1}(1-s)^{\nu-\alpha-1}-(t-s)^{\nu-1}}{\Gamma(\nu)}, \quad 0 \leq s \leq t \leq 1, \\
\frac{t^{\nu-1}(1-s)^{\nu-\alpha-1}}{\Gamma(\nu)}, \quad 0 \leq t \leq s \leq 1,
\end{array}\right.
$$

is the Green function for this problem.

Lemma 2.3 (See [19]) Let $G(t, s)$ be as given in the statement of Lemma 2.2. Then we have

(i) $G(t, s)$ is a continuous function on the unit square $[0,1] \times[0,1]$;

(ii) $G(t, s) \geq 0$ for each $(t, s) \in[0,1] \times[0,1]$.

Lemma 2.4 The function $G(t, s)$ defined by (2.2) satisfies the following conditions:

$$
\left[1-(1-s)^{\alpha}\right](1-s)^{\nu-\alpha-1} t^{\nu-1} \leq \Gamma(\nu) G(t, s) \leq(1-s)^{\nu-\alpha-1} t^{\nu-1}, \quad t, s \in[0,1] .
$$

Proof Evidently, the right inequality holds. So, we only need to prove the left inequality. If $0 \leq s \leq t \leq 1$, then we have $0 \leq t-s \leq t-t s=(1-s) t$, and thus

$$
(t-s)^{\nu-1} \leq(1-s)^{\nu-1} t^{\nu-1}
$$

Hence,

$$
\begin{aligned}
\Gamma(v) G(t, s) & =t^{\nu-1}(1-s)^{\nu-\alpha-1}-(t-s)^{\nu-1} \\
& \geq t^{\nu-1}(1-s)^{\nu-\alpha-1}-t^{\nu-1}(1-s)^{\nu-1}
\end{aligned}
$$




$$
\begin{aligned}
& =t^{\nu-1}\left[(1-s)^{\nu-\alpha-1}-(1-s)^{\nu-1}\right] \\
& =\left[1-(1-s)^{\alpha}\right](1-s)^{\nu-\alpha-1} t^{\nu-1} .
\end{aligned}
$$

When $0 \leq t \leq s \leq 1$, we have

$$
\begin{aligned}
\Gamma(v) G(t, s) & =t^{\nu-1}(1-s)^{\nu-\alpha-1} \\
& \geq t^{\nu-1}\left[(1-s)^{\nu-\alpha-1}-(1-s)^{\nu-1}\right] \\
& =\left[1-(1-s)^{\alpha}\right](1-s)^{\nu-\alpha-1} t^{\nu-1} .
\end{aligned}
$$

So, the proof is complete.

In the sequel, we present some basic concepts in ordered Banach spaces for completeness and two fixed point theorems which we will be used later. For convenience of readers, we suggest that one refers to [20-22] for details.

Suppose that $(E,\|\cdot\|)$ is a real Banach space which is partially ordered by a cone $P \subset E$, i.e., $x \leq y$ if and only if $y-x \in P$. If $x \leq y$ and $x \neq y$, then we denote $x<y$ or $y>x$. By $\theta$ we denote the zero element of $E$. Recall that a non-empty closed convex set $P \subset E$ is a cone if it satisfies (i) $x \in P, \lambda \geq 0 \Rightarrow \lambda x \in P$; (ii) $x \in P,-x \in P \Rightarrow x=\theta$.

$P$ is called normal if there exists a constant $N>0$ such that, for all $x, y \in E, \theta \leq x \leq y$ implies $\|x\| \leq N\|y\|$; in this case, $N$ is called the normality constant of $P$. If $x_{1}, x_{2} \in E$, the set $\left[x_{1}, x_{2}\right]=\left\{x \in E \mid x_{1} \leq x \leq x_{2}\right\}$ is called the order interval between $x_{1}$ and $x_{2}$. We say that an operator $A: E \rightarrow E$ is increasing (decreasing) if $x \leq y$ implies $A x \leq A y(A x \geq A y)$.

For all $x, y \in E$, the notation $x \sim y$ means that there exist $\lambda>0$ and $\mu>0$ such that $\lambda x \leq y \leq \mu x$. Clearly, $\sim$ is an equivalence relation. Given $h>\theta$ (i.e., $h \geq \theta$ and $h \neq \theta$ ), we denote by $P_{h}$ the set $P_{h}=\{x \in E \mid x \sim h\}$. It is easy to see that $P_{h} \subset P$.

Definition 2.5 (See [20,22]) $A: P \times P \rightarrow P$ is said to be a mixed monotone operator if $A(x, y)$ is increasing in $x$ and decreasing in $y$, i.e., $u_{i}, v_{i}(i=1,2) \in P, u_{1} \leq u_{2}, v_{1} \geq v_{2}$ imply $A\left(u_{1}, v_{1}\right) \leq A\left(u_{2}, v_{2}\right)$. Element $x \in P$ is called a fixed point of $A$ if $A(x, x)=x$.

Definition 2.6 An operator $A: P \rightarrow P$ is said to be sub-homogeneous if it is satisfies

$$
A(t x) \geq t A(x), \quad \forall t \in(0,1), x \in P .
$$

Definition 2.7 Let $D=P$ and $\beta$ be a real number with $0 \leq \beta<1$. An operator $A: D \rightarrow D$ is said to be $\beta$-concave if it satisfies

$$
A(t x) \geq t^{\beta} A(x), \quad \forall t \in(0,1), x \in D .
$$

Lemma 2.8 (See Theorem 2.1 in [20]) Let $h>\theta$ and $\beta \in(0,1) . A: P \times P \rightarrow P$ is a mixed monotone operator and satisfies

$$
A\left(t x, t^{-1} y\right) \geq t^{\beta} A(x, y), \quad \forall t \in(0,1), x, y \in P .
$$

$B: P \rightarrow P$ is an increasing sub-homogeneous operator. Assume that

(i) there is $h_{0} \in P_{h}$ such that $A\left(h_{0}, h_{0}\right) \in P_{h}$ and $B h_{0} \in P_{h}$;

(ii) there exists a constant $\delta_{0}>0$ such that $A(x, y) \geq \delta_{0} B x, \forall x, y \in P$. 
Then:

(1) $A: P_{h} \times P_{h} \rightarrow P_{h}$ and $B: P_{h} \rightarrow P_{h}$;

(2) there exist $u_{0}, v_{0} \in P_{h}$ and $r \in(0,1)$ such that

$$
r v_{0} \leq u_{0}<v_{0}, \quad u_{0} \leq A\left(u_{0}, v_{0}\right)+B u_{0} \leq A\left(v_{0}, u_{0}\right)+B v_{0} \leq v_{0}
$$

(3) the operator equation $A(x, x)+B x=x$ has a unique solution $x^{*}$ in $P_{h}$;

(4) for any initial values $x_{0}, y_{0} \in P_{h}$, constructing successively the sequences

$$
x_{n}=A\left(x_{n-1}, y_{n-1}\right)+B x_{n-1}, \quad y_{n}=A\left(y_{n-1}, x_{n-1}\right)+B y_{n-1}, \quad n=1,2, \ldots,
$$

we have $x_{n} \rightarrow x^{*}$ and $y_{n} \rightarrow x^{*}$ as $n \rightarrow \infty$.

Lemma 2.9 (See Theorem 2.4 in [20]) Let $h>\theta$ and $\beta \in(0,1) . A: P \times P \rightarrow P$ is a mixed monotone operator and satisfies

$$
A\left(t x, t^{-1} y\right) \geq t A(x, y), \quad \forall t \in(0,1), x, y \in P .
$$

\section{$B: P \rightarrow P$ is an increasing $\beta$-concave operator. Assume that}

(i) there is $h_{0} \in P_{h}$ such that $A\left(h_{0}, h_{0}\right) \in P_{h}$ and $B h_{0} \in P_{h}$;

(ii) there exists a constant $\delta_{0}>0$ such that $A(x, y) \leq \delta_{0} B x, \forall x, y \in P$.

Then:

(1) $A: P_{h} \times P_{h} \rightarrow P_{h}$ and $B: P_{h} \rightarrow P_{h}$;

(2) there exist $u_{0}, v_{0} \in P_{h}$ and $r \in(0,1)$ such that

$$
r v_{0} \leq u_{0}<v_{0}, \quad u_{0} \leq A\left(u_{0}, v_{0}\right)+B u_{0} \leq A\left(v_{0}, u_{0}\right)+B v_{0} \leq v_{0} ;
$$

(3) the operator equation $A(x, x)+B x=x$ has a unique solution $x^{*}$ in $P_{h}$;

(4) for any initial values $x_{0}, y_{0} \in P_{h}$, constructing successively the sequences

$$
x_{n}=A\left(x_{n-1}, y_{n-1}\right)+B x_{n-1}, \quad y_{n}=A\left(y_{n-1}, x_{n-1}\right)+B y_{n-1}, \quad n=1,2, \ldots,
$$

we have $x_{n} \rightarrow x^{*}$ and $y_{n} \rightarrow x^{*}$ as $n \rightarrow \infty$.

Remark 2.10 (i) If we take $B=\theta$ in Lemma 2.8, then the corresponding conclusion is still true (see Corollary 2.2 in [20]); (ii) if we take $A=\theta$ in Lemma 2.9, then the conclusion obtained is also true (see Theorem 2.7 in [23]).

\section{Main results}

In this section, we apply Lemma 2.8 and Lemma 2.9 to study FBVP (1.1), and we obtain some new results on the existence and uniqueness of positive solutions. The method used here is relatively new to the literature and so are the existence and uniqueness results to the fractional differential equations.

In our considerations, we work in the Banach space $C[0,1]=\{x:[0,1] \rightarrow \mathbf{R}$ is continuous $\}$ with the standard norm $\|x\|=\sup \{|x(t)|: t \in[0,1]\}$. Notice that this space can be equipped with a partial order given by

$$
x, y \in C[0,1], \quad x \leq y \quad \Leftrightarrow \quad x(t) \leq y(t) \quad \text { for } t \in[0,1] .
$$


Set $P=\{x \in C[0,1] \mid x(t) \geq 0, t \in[0,1]\}$, the standard cone. It is clear that $P$ is a normal cone in $C[0,1]$ and the normality constant is 1 .

\section{Theorem 3.1 Assume that}

$\left(\mathrm{H}_{1}\right) f:[0,1] \times[0,+\infty) \times[0,+\infty) \rightarrow[0,+\infty)$ is continuous and $g:[0,1] \times[0,+\infty) \rightarrow$ $[0,+\infty)$ is continuous;

$\left(\mathrm{H}_{2}\right) f(t, u, v)$ is increasing in $u \in[0,+\infty)$ for fixed $t \in[0,1]$ and $v \in[0,+\infty)$, decreasing in $v \in[0,+\infty)$ for fixed $t \in[0,1]$ and $u \in[0,+\infty)$, and $g(t, u)$ is increasing in $u \in[0,+\infty)$ for fixed $t \in[0,1]$

$\left(\mathrm{H}_{3}\right) g(t, 0) \not \equiv 0$ and $g(t, \lambda u) \geq \lambda g(t, u)$ for $\lambda \in(0,1), t \in[0,1], u \in[0,+\infty)$, and there exists a constant $\beta \in(0,1)$ such that $f\left(t, \lambda u, \lambda^{-1} v\right) \geq \lambda^{\beta} f(t, u, v), \forall t \in[0,1], \lambda \in(0,1), u, v \in$ $[0,+\infty)$;

$\left(\mathrm{H}_{4}\right)$ there exists a constant $\delta_{0}>0$ such that $f(t, u, v) \geq \delta_{0} g(t, u), t \in[0,1], u, v \geq 0$.

Then:

(1) there exist $u_{0}, v_{0} \in P_{h}$ and $r \in(0,1)$ such that $r v_{0} \leq u_{0}<v_{0}$ and

$$
\begin{aligned}
& u_{0}(t) \leq \int_{0}^{1} G(t, s)\left[f\left(s, u_{0}(s), v_{0}(s)\right)+g\left(s, u_{0}(s)\right)\right] d s, \quad t \in[0,1], \\
& v_{0}(t) \geq \int_{0}^{1} G(t, s)\left[f\left(s, v_{0}(s), u_{0}(s)\right)+g\left(s, v_{0}(s)\right)\right] d s, \quad t \in[0,1]
\end{aligned}
$$

where $h(t)=t^{\nu-1}, t \in[0,1]$ and $G(t, s)$ is given as in (2.2);

(2) FBVP (1.1) has a unique positive solution $u^{*}$ in $P_{h}$;

(3) for any $x_{0}, y_{0} \in P_{h}$, constructing successively the sequences

$$
\begin{aligned}
& x_{n+1}(t)=\int_{0}^{1} G(t, s)\left[f\left(s, x_{n}(s), y_{n}(s)\right)+g\left(s, x_{n}(s)\right)\right] d s, \quad n=0,1,2, \ldots, \\
& y_{n+1}(t)=\int_{0}^{1} G(t, s)\left[f\left(s, y_{n}(s), x_{n}(s)\right)+g\left(s, y_{n}(s)\right)\right] d s, \quad n=0,1,2, \ldots,
\end{aligned}
$$

we have $\left\|x_{n}-u^{*}\right\| \rightarrow 0$ and $\left\|y_{n}-u^{*}\right\| \rightarrow 0$ as $n \rightarrow \infty$.

Proof To begin with, from Lemma 2.2, FBVP (1.1) has an integral formulation given by

$$
u(t)=\int_{0}^{1} G(t, s)[f(s, u(s), u(s))+g(s, u(s))] d s
$$

where $G(t, s)$ is given as in (2.2).

Define two operators $A: P \times P \rightarrow E$ and $B: P \rightarrow E$ by

$$
A(u, v)(t)=\int_{0}^{1} G(t, s) f(s, u(s), v(s)) d s, \quad(B u)(t)=\int_{0}^{1} G(t, s) g(s, u(s)) d s
$$

It is easy to prove that $u$ is the solution of FBVP (1.1) if and only if $u=A(u, u)+B u$. From $\left(\mathrm{H}_{1}\right)$, we know that $A: P \times P \rightarrow P$ and $B: P \rightarrow P$. In the sequel, we check that $A$, $B$ satisfy all the assumptions of Lemma 2.8 . 
Firstly, we prove that $A$ is a mixed monotone operator. In fact, for $u_{i}, v_{i} \in P, i=1,2$ with $u_{1} \geq u_{2}, v_{1} \leq v_{2}$, we know that $u_{1}(t) \geq u_{2}(t), v_{1}(t) \leq v_{2}(t), t \in[0,1]$, and by $\left(\mathrm{H}_{2}\right)$ and Lemma 2.3,

$$
A\left(u_{1}, v_{1}\right)(t)=\int_{0}^{1} G(t, s) f\left(s, u_{1}(s), v_{1}(s)\right) d s \geq \int_{0}^{1} G(t, s) f\left(s, u_{2}(s), v_{2}(s)\right) d s=A\left(u_{2}, v_{2}\right)(t) .
$$

That is, $A\left(u_{1}, v_{1}\right) \geq A\left(u_{2}, v_{2}\right)$.

Further, it follows from $\left(\mathrm{H}_{2}\right)$ and Lemma 2.3 that $B$ is increasing. Next we show that $A$ satisfies the condition (2.5). For any $\lambda \in(0,1)$ and $u, v \in P$, by $\left(\mathrm{H}_{3}\right)$ we have

$$
\begin{aligned}
A\left(\lambda u, \lambda^{-1} v\right)(t) & =\int_{0}^{1} G(t, s) f\left(s, \lambda u(s), \lambda^{-1} v(s)\right) d s \\
& \geq \lambda^{\beta} \int_{0}^{1} G(t, s) f(s, u(s), v(s)) d s \\
& =\lambda^{\beta} A(u, v)(t) .
\end{aligned}
$$

That is, $A\left(\lambda u, \lambda^{-1} v\right) \geq \lambda^{\beta} A(u, v)$ for $\lambda \in(0,1), u, v \in P$. So, the operator $A$ satisfies (2.5). Also, for any $\lambda \in(0,1), u \in P$, from $\left(\mathrm{H}_{3}\right)$ we know that

$$
B(\lambda u)(t)=\int_{0}^{1} G(t, s) g(s, \lambda u(s)) d s \geq \lambda \int_{0}^{1} G(t, s) g(s, u(s)) d s=\lambda B u(t),
$$

that is, $B(\lambda u) \geq \lambda B u$ for $\lambda \in(0,1), u \in P$. That is, the operator $B$ is sub-homogeneous. Now we show that $A(h, h) \in P_{h}$ and $B h \in P_{h}$. On the one hand, from $\left(\mathrm{H}_{1}\right),\left(\mathrm{H}_{2}\right)$ and Lemma 2.4, for any $t \in[0,1]$, we have

$$
\begin{aligned}
A(h, h)(t) & =\int_{0}^{1} G(t, s) f(s, h(s), h(s)) d s \\
& =\int_{0}^{1} G(t, s) f\left(s, s^{\nu-1}, s^{\nu-1}\right) d s \\
& \leq \frac{1}{\Gamma(\nu)} h(t) \int_{0}^{1}(1-s)^{\nu-\alpha-1} f(s, 1,0) d s .
\end{aligned}
$$

On the other hand, also from $\left(\mathrm{H}_{1}\right),\left(\mathrm{H}_{2}\right)$ and Lemma 2.4, for any $t \in[0,1]$, we obtain

$$
\begin{aligned}
A(h, h)(t) & =\int_{0}^{1} G(t, s) f(s, h(s), h(s)) d s \\
& =\int_{0}^{1} G(t, s) f\left(s, s^{\nu-1}, s^{\nu-1}\right) d s \\
& \geq \frac{1}{\Gamma(v)} h(t) \int_{0}^{1}\left[1-(1-s)^{\alpha}\right](1-s)^{\nu-\alpha-1} f(s, 0,1) d s .
\end{aligned}
$$

From $\left(\mathrm{H}_{2}\right),\left(\mathrm{H}_{4}\right)$, we have

$$
f(s, 1,0) \geq f(s, 0,1) \geq \delta_{0} g(s, 0) \geq 0 .
$$


Since $g(t, 0) \not \equiv 0$, we get

$$
\int_{0}^{1} f(s, 1,0) d s \geq \int_{0}^{1} f(s, 0,1) d s \geq \delta_{0} \int_{0}^{1} g(s, 0) d s>0,
$$

and in consequence,

$$
\begin{aligned}
& l_{1}:=\frac{1}{\Gamma(v)} \int_{0}^{1}(1-s)^{\nu-\alpha-1} f(s, 1,0) d s>0, \\
& l_{2}:=\frac{1}{\Gamma(\nu)} \int_{0}^{1}\left[1-(1-s)^{\alpha}\right](1-s)^{\nu-\alpha-1} f(s, 0,1) d s>0 .
\end{aligned}
$$

So, $l_{2} h(t) \leq A(h, h)(t) \leq l_{1} h(t), t \in[0,1]$; and hence we have $A(h, h) \in P_{h}$. Similarly,

$$
\frac{1}{\Gamma(\nu)} h(t) \int_{0}^{1}\left[1-(1-s)^{\alpha}\right](1-s)^{\nu-\alpha-1} g(s, 0) d s \leq B h(t) \leq \frac{1}{\Gamma(\nu)} h(t) \int_{0}^{1}(1-s)^{\nu-\alpha-1} g(s, 1) d s,
$$

from $g(t, 0) \not \equiv 0$, we easily prove $B h \in P_{h}$. Hence the condition (i) of Lemma 2.8 is satisfied.

In the following, we show the condition (ii) of Lemma 2.8 is satisfied. For $u, v \in P$, and any $t \in[0,1]$, from $\left(\mathrm{H}_{4}\right)$,

$$
A(u, v)(t)=\int_{0}^{1} G(t, s) f(s, u(s), v(s)) d s \geq \delta_{0} \int_{0}^{1} G(t, s) g(s, u(s)) d s=\delta_{0} B u(t) .
$$

Then we get $A(u, v) \geq \delta_{0} B u$, for $u, v \in P$. Finally, an application of Lemma 2.8 implies: there exist $u_{0}, v_{0} \in P_{h}$ and $r \in(0,1)$ such that $r v_{0} \leq u_{0}<v_{0}, u_{0} \leq A\left(u_{0}, v_{0}\right)+B u_{0} \leq A\left(v_{0}, u_{0}\right)+$ $B v_{0} \leq v_{0}$; the operator equation $A(u, u)+B u=u$ has a unique solution $u^{*}$ in $P_{h}$; for any initial values $x_{0}, y_{0} \in P_{h}$, constructing successively the sequences

$$
x_{n}=A\left(x_{n-1}, y_{n-1}\right)+B x_{n-1}, \quad y_{n}=A\left(y_{n-1}, x_{n-1}\right)+B y_{n-1}, \quad n=1,2, \ldots,
$$

we have $x_{n} \rightarrow u^{*}$ and $y_{n} \rightarrow u^{*}$ as $n \rightarrow \infty$. That is,

$$
\begin{aligned}
& u_{0}(t) \leq \int_{0}^{1} G(t, s)\left[f\left(s, u_{0}(s), v_{0}(s)\right)+g\left(s, u_{0}(s)\right)\right] d s, \quad t \in[0,1], \\
& v_{0}(t) \geq \int_{0}^{1} G(t, s)\left[f\left(s, v_{0}(s), u_{0}(s)\right)+g\left(s, v_{0}(s)\right)\right] d s, \quad t \in[0,1]
\end{aligned}
$$

FBVP (1.1) has a unique positive solution $u^{*}$ in $P_{h}$; for $x_{0}, y_{0} \in P_{h}$, the sequences

$$
\begin{aligned}
& x_{n+1}(t)=\int_{0}^{1} G(t, s)\left[f\left(s, x_{n}(s), y_{n}(s)\right)+g\left(s, x_{n}(s)\right)\right] d s, \quad n=0,1,2, \ldots, \\
& y_{n+1}(t)=\int_{0}^{1} G(t, s)\left[f\left(s, y_{n}(s), x_{n}(s)\right)+g\left(s, y_{n}(s)\right)\right] d s, \quad n=0,1,2, \ldots,
\end{aligned}
$$

satisfy $\left\|x_{n}-u^{*}\right\| \rightarrow 0$ and $\left\|y_{n}-u^{*}\right\| \rightarrow 0$ as $n \rightarrow \infty$.

Theorem 3.2 Assume $\left(\mathrm{H}_{1}\right),\left(\mathrm{H}_{2}\right)$ and

$\left(\mathrm{H}_{5}\right)$ there exists a constant $\beta \in(0,1)$ such that $g(t, \lambda u) \geq \lambda^{\beta} g(t, u), \forall t \in[0,1], \lambda \in(0,1)$, $u \in[0,+\infty)$, and $f\left(t, \lambda u, \lambda^{-1} v\right) \geq \lambda f(t, u, v)$ for $\lambda \in(0,1), t \in[0,1], u, v \in[0,+\infty)$; 
$\left(\mathrm{H}_{6}\right) f(t, 0,1) \not \equiv 0$ for $t \in[0,1]$ and there exists a constant $\delta_{0}>0$ such that $f(t, u, v) \leq$ $\delta_{0} g(t, u), t \in[0,1], u, v \geq 0$.

Then:

(1) there exist $u_{0}, v_{0} \in P_{h}$ and $r \in(0,1)$ such that $r v_{0} \leq u_{0}<v_{0}$ and

$$
\begin{aligned}
& u_{0}(t) \leq \int_{0}^{1} G(t, s)\left[f\left(s, u_{0}(s), v_{0}(s)\right)+g\left(s, u_{0}(s)\right)\right] d s, \quad t \in[0,1], \\
& v_{0}(t) \geq \int_{0}^{1} G(t, s)\left[f\left(s, v_{0}(s), u_{0}(s)\right)+g\left(s, v_{0}(s)\right)\right] d s, \quad t \in[0,1],
\end{aligned}
$$

where $h(t)=t^{\nu-1}, t \in[0,1]$ and $G(t, s)$ is given as in (2.2);

(2) FBVP (1.1) has a unique positive solution $u^{*}$ in $P_{h}$;

(3) for any $x_{0}, y_{0} \in P_{h}$, constructing successively the sequences

$$
\begin{array}{ll}
x_{n+1}(t)=\int_{0}^{1} G(t, s)\left[f\left(s, x_{n}(s), y_{n}(s)\right)+g\left(s, x_{n}(s)\right)\right] d s, & n=0,1,2, \ldots, \\
y_{n+1}(t)=\int_{0}^{1} G(t, s)\left[f\left(s, y_{n}(s), x_{n}(s)\right)+g\left(s, y_{n}(s)\right)\right] d s, \quad n=0,1,2, \ldots,
\end{array}
$$

we have $\left\|x_{n}-u^{*}\right\| \rightarrow 0$ and $\left\|y_{n}-u^{*}\right\| \rightarrow 0$ as $n \rightarrow \infty$.

Sketch of the proof Consider two operators $A, B$ defined in the proof of Theorem 3.1. Similarly, from $\left(\mathrm{H}_{1}\right),\left(\mathrm{H}_{2}\right)$, we obtain that $A: P \times P \rightarrow P$ is a mixed monotone operator and $B: P \rightarrow P$ is increasing. From $\left(\mathrm{H}_{5}\right)$, we have

$$
A\left(\lambda u, \lambda^{-1} v\right) \geq \lambda A(u, v) ; \quad B(\lambda u) \geq \lambda^{\beta} B u, \quad \text { for } \lambda \in(0,1), u, v \in P .
$$

From $\left(\mathrm{H}_{2}\right),\left(\mathrm{H}_{6}\right)$, we have

$$
g(s, 0) \geq \frac{1}{\delta_{0}} f(s, 0,1), \quad f(s, 1,0) \geq f(s, 0,1), \quad s \in[0,1] .
$$

Since $f(t, 0,1) \not \equiv 0$, we get

$$
\begin{aligned}
& \int_{0}^{1} f(s, 1,0) d s \geq \int_{0}^{1} f(s, 0,1) d s>0, \\
& \int_{0}^{1} g(s, 1) d s \geq \int_{0}^{1} g(s, 0) d s \geq \frac{1}{\delta_{0}} \int_{0}^{1} f(s, 0,1) d s>0,
\end{aligned}
$$

and in consequence,

$$
\begin{aligned}
& \frac{1}{\Gamma(\nu)} \int_{0}^{1}(1-s)^{\nu-\alpha-1} f(s, 1,0) d s \geq \frac{1}{\Gamma(\nu)} \int_{0}^{1}\left[1-(1-s)^{\alpha}\right](1-s)^{\nu-\alpha-1} f(s, 0,1) d s>0, \\
& \frac{1}{\Gamma(\nu)} \int_{0}^{1}(1-s)^{\nu-\alpha-1} g(s, 1) d s \geq \frac{1}{\Gamma(\nu)} \int_{0}^{1}\left[1-(1-s)^{\alpha}\right](1-s)^{\nu-\alpha-1} g(s, 0) d s>0 .
\end{aligned}
$$

So, we can easily prove that $A(h, h) \in P_{h}, B h \in P_{h}$. For $u, v \in P$, and any $t \in[0,1]$, from $\left(\mathrm{H}_{6}\right)$,

$$
A(u, v)(t)=\int_{0}^{1} G(t, s) f(s, u(s), v(s)) d s \leq \delta_{0} \int_{0}^{1} G(t, s) g(s, u(s)) d s=\delta_{0} B u(t)
$$


Then we get $A(u, v) \leq \delta_{0} B u$, for $u, v \in P$. Finally, an application of Lemma 2.9 implies: there exist $u_{0}, v_{0} \in P_{h}$ and $r \in(0,1)$ such that $r v_{0} \leq u_{0}<v_{0}, u_{0} \leq A\left(u_{0}, v_{0}\right)+B u_{0} \leq A\left(v_{0}, u_{0}\right)+$ $B v_{0} \leq v_{0}$; the operator equation $A(u, u)+B u=u$ has a unique solution $u^{*}$ in $P_{h}$; for any initial values $x_{0}, y_{0} \in P_{h}$, constructing successively the sequences

$$
x_{n}=A\left(x_{n-1}, y_{n-1}\right)+B x_{n-1}, \quad y_{n}=A\left(y_{n-1}, x_{n-1}\right)+B y_{n-1}, \quad n=1,2, \ldots,
$$

we have $x_{n} \rightarrow u^{*}$ and $y_{n} \rightarrow u^{*}$ as $n \rightarrow \infty$. That is,

$$
\begin{array}{ll}
u_{0}(t) \leq \int_{0}^{1} G(t, s)\left[f\left(s, u_{0}(s), v_{0}(s)\right)+g\left(s, u_{0}(s)\right)\right] d s, & t \in[0,1], \\
v_{0}(t) \geq \int_{0}^{1} G(t, s)\left[f\left(s, v_{0}(s), u_{0}(s)\right)+g\left(s, v_{0}(s)\right)\right] d s, & t \in[0,1] ;
\end{array}
$$

FBVP (1.1) has a unique positive solution $u^{*}$ in $P_{h}$; for $x_{0}, y_{0} \in P_{h}$, the sequences

$$
\begin{array}{ll}
x_{n+1}(t)=\int_{0}^{1} G(t, s)\left[f\left(s, x_{n}(s), y_{n}(s)\right)+g\left(s, x_{n}(s)\right)\right] d s, & n=0,1,2, \ldots, \\
y_{n+1}(t)=\int_{0}^{1} G(t, s)\left[f\left(s, y_{n}(s), x_{n}(s)\right)+g\left(s, y_{n}(s)\right)\right] d s, & n=0,1,2, \ldots,
\end{array}
$$

satisfy $\left\|x_{n}-u^{*}\right\| \rightarrow 0$ and $\left\|y_{n}-u^{*}\right\| \rightarrow 0$ as $n \rightarrow \infty$.

From Remark 2.10 and similar to the proofs of Theorems 3.1-3.2, we can prove the following conclusions.

Corollary 3.3 Let $g \equiv 0$. Assume that $f$ satisfies the conditions of Theorem 3.1 and $f(t, 0,1) \not \equiv 0$. Then: (i) there exist $u_{0}, v_{0} \in P_{h}$ and $r \in(0,1)$ such that $r v_{0} \leq u_{0}<v_{0}$ and

$$
\begin{aligned}
& u_{0}(t) \leq \int_{0}^{1} G(t, s) f\left(s, u_{0}(s), v_{0}(s)\right) d s, \\
& v_{0}(t) \geq \int_{0}^{1} G(t, s) f\left(s, v_{0}(s), u_{0}(s)\right) d s, \quad t \in[0,1]
\end{aligned}
$$

where $h(t)=t^{\nu-1}, t \in[0,1]$ and $G(t, s)$ is given as in (2.2); (ii) the FBVP

$$
\left\{\begin{array}{l}
-D_{0^{+}}^{v} y(t)=f(t, y(t), y(t)), \quad 0<t<1, n-1<v \leq n, \\
y^{(i)}(0)=0, \quad 0 \leq i \leq n-2, \\
{\left[D_{0^{+}}^{\alpha} y(t)\right]_{t=1}=0, \quad 1 \leq \alpha \leq n-2,}
\end{array}\right.
$$

has a unique positive solution $u^{*}$ in $P_{h}$; (iii) for any $x_{0}, y_{0} \in P_{h}$, constructing successively the sequences

$$
\begin{aligned}
& x_{n+1}(t)=\int_{0}^{1} G(t, s) f\left(s, x_{n}(s), y_{n}(s)\right) d s, \\
& y_{n+1}(t)=\int_{0}^{1} G(t, s) f\left(s, y_{n}(s), x_{n}(s)\right) d s, \quad n=0,1,2, \ldots,
\end{aligned}
$$

we have $\left\|x_{n}-u^{*}\right\| \rightarrow 0$ and $\left\|y_{n}-u^{*}\right\| \rightarrow 0$ as $n \rightarrow \infty$. 
Corollary 3.4 Let $f \equiv 0$. Assume that $g$ satisfies the conditions of Theorem 3.2 and $g(t, 0) \not \equiv$ 0 for $t \in[0,1]$. Then: (i) there exist $u_{0}, v_{0} \in P_{h}$ and $r \in(0,1)$ such that $r v_{0} \leq u_{0}<v_{0}$ and

$$
u_{0}(t) \leq \int_{0}^{1} G(t, s) g\left(s, u_{0}(s)\right) d s, \quad v_{0}(t) \geq \int_{0}^{1} G(t, s) g\left(s, v_{0}(s)\right) d s, \quad t \in[0,1],
$$

where $h(t)=t^{\nu-1}, t \in[0,1]$ and $G(t, s)$ is given as in (2.2); (ii) the FBVP

$$
\left\{\begin{array}{l}
-D_{0^{+}}^{v} y(t)=g(t, y(t)), \quad 0<t<1, n-1<v \leq n \\
y^{(i)}(0)=0, \quad 0 \leq i \leq n-2 \\
{\left[D_{0^{+}}^{\alpha} y(t)\right]_{t=1}=0, \quad 1 \leq \alpha \leq n-2}
\end{array}\right.
$$

has a unique positive solution $u^{*}$ in $P_{h}$; (iii) for any $x_{0}, y_{0} \in P_{h}$, constructing successively the sequences

$$
x_{n+1}(t)=\int_{0}^{1} G(t, s) g\left(s, x_{n}(s)\right) d s, \quad y_{n+1}(t)=\int_{0}^{1} G(t, s) g\left(s, y_{n}(s)\right) d s, \quad n=0,1,2, \ldots,
$$

we have $\left\|x_{n}-u^{*}\right\| \rightarrow 0$ and $\left\|y_{n}-u^{*}\right\| \rightarrow 0$ as $n \rightarrow \infty$.

\section{An example}

We now present one example to illustrate Theorem 3.1.

Example 4.1 Consider the following FBVP:

$$
\left\{\begin{array}{l}
-D_{0^{+}}^{6.3} u(t)=u^{\frac{1}{4}}(t)+[u(t)+2]^{-\frac{1}{3}}+\frac{u(t)}{1+u(t)} a(t)+b(t)+c, \quad 0<t<1 \\
u^{(i)}(0)=0, \quad 0 \leq i \leq 5 \\
{\left[D_{0^{+}}^{4.2} u(t)\right]_{t=1}=0}
\end{array}\right.
$$

where $c>0$ is a constant, $a, b:[0,1] \rightarrow[0, \infty)$ are continuous with $a \neq 0$.

Obviously, problem (4.1) fits the framework of FBVP (1.1) with $v=6.3, \alpha=4.2$. (Note that $n=7$, therefore, in this case.) In this example, we take $0<d<c$ and let

$$
\begin{aligned}
& f(t, u, v)=u^{\frac{1}{4}}+[v+2]^{-\frac{1}{3}}+b(t)+d, \quad g(t, u)=\frac{u}{1+u} a(t)+c-d, \\
& \beta=\frac{1}{3}, \quad a_{\max }=\max \{a(t): t \in[0,1]\} .
\end{aligned}
$$

Obviously, $a_{\max }>0 ; f:[0,1] \times[0,+\infty) \times[0,+\infty) \rightarrow[0,+\infty)$ is continuous and $g:[0,1] \times$ $[0,+\infty) \rightarrow[0,+\infty)$ is continuous with $g(t, 0)=c-d>0$. And $f(t, u, v)$ is increasing in $u \in[0,+\infty)$ for fixed $t \in[0,1]$ and $v \in[0,+\infty)$, decreasing in $v \in[0,+\infty)$ for fixed $t \in[0,1]$ and $u \in[0,+\infty)$, and $g(t, u)$ is increasing in $u \in[0,+\infty)$ for fixed $t \in[0,1]$. Besides, for $\lambda \in(0,1), t \in[0,1], u \in[0, \infty)$, we have

$$
\begin{gathered}
g(t, \lambda u)=\frac{\lambda u}{1+\lambda u} a(t)+c-d \geq \frac{\lambda u}{1+u} a(t)+\lambda(c-d)=\lambda g(t, u), \\
\begin{aligned}
f\left(t, \lambda u, \lambda^{-1} v\right) & =\lambda^{\frac{1}{4}} u^{\frac{1}{4}}+\lambda^{\frac{1}{3}}[v+2 \lambda]^{-\frac{1}{3}}+b(t)+d \\
& \geq \lambda^{\frac{1}{3}}\left\{u^{\frac{1}{4}}+[v+2]^{-\frac{1}{3}}+b(t)+d\right\}=\lambda^{\beta} f(t, u, v) .
\end{aligned}
\end{gathered}
$$


Moreover, if we take $\delta_{0} \in\left(0, \frac{d}{a_{\max }+c-d}\right]$, then we obtain

$$
\begin{aligned}
f(t, u, v) & =u^{\frac{1}{4}}+[v+2]^{-\frac{1}{3}}+b(t)+d \geq d=\frac{d}{a_{\max }+c-d} \cdot\left(a_{\max }+c-d\right) \\
& \geq \delta_{0}\left[\frac{u}{1+u} a(t)+c-d\right]=\delta_{0} g(t, u)
\end{aligned}
$$

Hence all the conditions of Theorem 3.1 are satisfied. An application of Theorem 3.1 implies that problem (4.1) has a unique positive solution in $P_{h}$, where $h(t)=t^{\nu-1}=t^{5.3}$, $t \in[0,1]$.

\section{Competing interests}

The authors declare that they have no competing interests.

\section{Authors' contributions}

The authors declare that the study was realized in collaboration with the same responsibility. All authors read and approved the final manuscript.

\section{Acknowledgements}

The authors are grateful to the anonymous referee for his/her valuable suggestions. The first author was supported financially by the Youth Science Foundations of China (11201272) and Shanxi Province (2010021002-1).

Received: 22 November 2012 Accepted: 18 March 2013 Published: 10 April 2013

\section{References}

1. Oldham, KB, Spanier, J: The Fractional Calculus. Academic Press, New York (1974)

2. Gaul, L, Klein, P, Kempffe, S: Damping description involving fractional operators. Mech. Syst. Signal Process. 5, 81-88 (1991)

3. Miller, KS, Ross, B: An Introduction to the Fractional Calculus and Fractional Differential Equations. Wiley, New York (1993)

4. Glockle, WG, Nonnenmacher, TF: A fractional calculus approach of self-similar protein dynamics. Biophys. J. 68, 46-53 (1995)

5. Podlubny, I: Fractional Differential Equations. Mathematics in Science and Engineering. Academic Press, New York (1999)

6. Kilbas, AA, Srivastava, HM, Trujillo, JJ: Theory and Applications of Fractional Differential Equations. North-Holland Mathematics Studies, vol. 204. Elsevier, Amsterdam (2006)

7. Zhang, SQ: Existence of positive solution for some class of nonlinear fractional differential equations. J. Math. Anal. Appl. 278, 136-148 (2003)

8. Bai, ZB, Lü, HS: Positive solutions for boundary value problem of nonlinear fractional differential equation. J. Math Anal. Appl. 311, 495-505 (2005)

9. Lakshmikantham, V: Theory of fractional functional differential equations. Nonlinear Anal. 69, 3337-3343 (2008)

10. Zhou, Y: Existence and uniqueness of fractional functional differential equations with unbounded delay. Int. J. Dyn. Syst. Differ. Equ. 1(4), 239-244 (2008)

11. Kaufmann, ER, Mboumi, E: Positive solutions of a boundary value problem for a nonlinear fractional differential equation. Electron. J. Qual. Theory Differ. Equ. 2008, 3 (2008)

12. Kosmatov, N: A singular boundary value problem for nonlinear differential equations of fractional order. J. Appl. Math. Comput. 29, 125-135 (2009)

13. $\mathrm{Xu}, \mathrm{X}$, Jiang, D, Yuan, C: Multiple positive solutions for boundary value problem of nonlinear fractional differential equation. Nonlinear Anal. 71, 4676-4688 (2009)

14. Yang, L, Chen, H: Unique positive solutions for fractional differential equation boundary value problems. Appl. Math. Lett. 23, 1095-1098 (2010)

15. Wang, YQ, Liu, LS, Wu, YH: Positive solutions for a nonlocal fractional differential equation. Nonlinear Anal. 74 , 3599-3605 (2011)

16. Lizama, C: An operator theoretical approach to a class of fractional order differential equations. Appl. Math. Lett. 24, 184-190 (2011)

17. Yang, C, Zhai, CB: Uniqueness of positive solutions for a fractional differential equation via a fixed point theorem of a sum operator. Electron. J. Differ. Equ. 2012, 70 (2012)

18. Goodrich, CS: Existence of a positive solution to a class of fractional differential equations. Appl. Math. Lett. 23, 1050-1055 (2010)

19. Goodrich, CS: Existence of a positive solution to systems of differential equations of fractional order. Comput. Math. Appl. 62, 1251-1268 (2011)

20. Zhai, CB, Hao, MR: Fixed point theorems for mixed monotone operators with perturbation and applications to fractional differential equation boundary value problems. Nonlinear Anal. 75, 2542-2551 (2012)

21. Guo, D, Lakshmikantham, V: Nonlinear Problems in Abstract Cones. Academic Press, Boston (1988) 
22. Guo, D, Lakskmikantham, V: Coupled fixed points of nonlinear operators with applications. Nonlinear Anal. 11(5), 623-632 (1987)

23. Zhai, CB, Yang, C, Zhang, XQ: Positive solutions for nonlinear operator equations and several classes of applications. Math. Z. 266, 43-63 (2010)

doi:10.1186/1687-2770-2013-85

Cite this article as: Zhai and Hao: Mixed monotone operator methods for the existence and uniqueness of positive solutions to Riemann-Liouville fractional differential equation boundary value problems. Boundary Value Problems $20132013: 85$.

Submit your manuscript to a SpringerOpen ${ }^{\odot}$ journal and benefit from:

- Convenient online submission

- Rigorous peer review

- Immediate publication on acceptance

Open access: articles freely available online

- High visibility within the field

- Retaining the copyright to your article

Submit your next manuscript at $\boldsymbol{s p r i n g e r o p e n . c o m ~}$ 